\title{
Metabolic engineering of Escherichia coli carrying the hybrid acetone-biosynthesis pathway for efficient acetone biosynthesis from acetate
}

\author{
Hao Yang ${ }^{1 \dagger}$, Bing Huang ${ }^{1 \dagger}$, Ningyu Lai ${ }^{1}$, Yang Gu${ }^{4}$, Zhimin $\mathrm{Li}^{1,2}$, Qin Ye ${ }^{1}$ and Hui Wu ${ }^{1,2,3^{*}}$ (D)
}

\begin{abstract}
Background: The shortage of food based feedstocks has been one of the stumbling blocks in industrial biomanufacturing. The acetone bioproduction from the traditional acetone-butanol-ethanol fermentation is limited by the nonspecificity of products and competitive utilization of food-based substrates. Using genetically modified Escherichia coli to produce acetone as sole product from the cost-effective non-food based substrates showed great potential to overcome these problems.
\end{abstract}

Results: A novel acetone biosynthetic pathway were constructed based on genes from Clostridium acetobutylicum (thlA encoding for thiolase, adc encoding for acetoacetate decarboxylase, ctfAB encoding for coenzyme A transferase) and Escherichia coli MG1655 (atoB encoding acetyl-CoA acetyltransferase, atoDA encoding for acetyl-CoA: acetoacetylCoA transferase subunit $\alpha$ and $\beta$ ). Among these constructs, one recombinant MG1655 derivative containing the hybrid pathway consisting of thIA, atoDA, and adc, produced the highest level of acetone from acetate. Reducing the gluconeogenesis pathway had little effect on acetone production, while blocking the TCA cycle by knocking out the $i c d A$ gene enhanced the yield of acetone significantly. As a result, acetone concentration increased up to $113.18 \mathrm{mM}$ in $24 \mathrm{~h}$ by the resting cell culture coupling with gas-stripping methods.

Conclusions: An engineered E. coli strain with optimized hybrid acetone biosynthetic pathway can utilize acetate as substrate efficiently to synthesize acetone without other non-gas byproducts. It provides a potential method for industrial biomanufacturing of acetone by engineered E. coli strains from non-food based substrate.

Keywords: Acetate, Acetone, Metabolic engineering, Escherichia coli, Gas-stripping, Resting cell

\section{Background}

Acetone is an important raw material for organic synthesis and a vital solvent in industry. At present, acetone is mainly produced as a co-product in the process of phenol production from cumene, which is a high efficiency and low cost process. However, the petrochemical routes are energy-consuming processes and depend on the

\footnotetext{
*Correspondence: hwu@ecust.edu.cn

${ }^{\dagger} \mathrm{Hao}$ Yang and Bing Huang contribute equally in this work

1 State Key Laboratory of Bioreactor Engineering, East China University of Science and Technology, 130 Meilong Road, Shanghai 200237, China Full list of author information is available at the end of the article
}

unrenewable fossil resources [1]. Bio-based industries has shown advantages over traditional fossil fuel based chemical industry on their environmental impact and resource sustainability. The acetone-butanol-ethanol $(\mathrm{ABE})$ fermentation is a classical acetone production via biological process, although some shortcomings are still existed in this fermentation process.

In the traditional $\mathrm{ABE}$ fermentation, the solvent-producing strains (such as Clostridium strains) usually use the food-based feedstocks (grain, maize, molasses and so on) as the substrates. Many efforts to improve the ratio and yield of butanol during the $\mathrm{ABE}$ fermentation were achieved by using different metabolic engineering 
strategies [2, 3]. However, a few improvements have been done to increase product specificity. The acetone biosynthetic pathway of C. acetobutylicum ATCC 824 was firstly introduced into $E$. coli and the engineered strain accumulated $40 \mathrm{mM}$ acetone in shake-flask culture supplying glucose as carbon source [4]. The CoA-transferase of acetone-synthesis cluster was replaced by one thioesterase which enabled the pathway independent of acetate or butyrate, resulting in $122 \mathrm{mM}$ acetone accumulation in glucose fed-batch culture [5]. A non-oxidative glycolysis pathway was also introduced into $E$. coli by genome expression phosphoketolase from Bifidobacterium adolescentis which improved the theoretical acetone yield from 1 to $1.5 \mathrm{~mol}$ acetone $/ \mathrm{mol}$ glucose and obtained $47 \mathrm{mM}$ acetone from glucose in shake-flasks [6]. These works improved the titer and theoretical yield of acetone production from glucose by engineered E. coli.

Nevertheless, due to the global food shortage and increase of food price, non-food based substrates, such as crude glycerol, methane, methanol and syngas, were developed as alternative substrates in bio-based industry [7-11]. Acetic acid, a cost-effective non-food based feedstock, can be generated from a variety of cheap sources via chemical or biological ways. A large proportion of acetic acid are produced chemically by liquidphase methanol carbonylation reaction [12]. Acetic acid also can be synthesized through syngas fermentation by Clostridium carboxidivorans [13], photosynthesis from $\mathrm{CO}_{2}$ by introducing the self-photosensitization into a nonphotosynthetic $M$. thermoacetica [14], and anaerobic acetogenesis by $M$. thermoacetica [15]. Moreover, acetic acid can also be recovered from lignocellulosic biomass hydrolyzates or pyrolyzates and industrial wastewater $[16,17]$. On the other hand, acetate utilization and acetyl-CoA metabolism in E. coli have been thoroughly studied, which makes acetic acid more feasible to be used as an alternate carbon. In recent years, acetate has been used to synthesize a series of value-added products, such as medium chain fatty acids [18], lipids [15], ethanol [19], itaconic acid [20], polyhydroxyalkanoates [21], mevalonate [22] and other acetyl-CoA derivatives. The CoA related acetate transportation in the acetone synthetic pathway of $C$. acetobutylicum made it a better substitution than other bio-pathways from acetate to acetone.

In this study, acetone was efficiently synthesized from acetate by constructing acetone synthetic pathway, enhancing acetate assimilation and manipulating central carbon metabolism in engineered $E$. coli. Four different combinations of acetone synthetic pathway were constructed, and their effect on acetone production were tested in E. coli MG1655, respectively. With the best acetone producer among them, ACK-PTA (acetate kinase and phosphotransacetylase) enzymes for acetate assimilation were overexpressed, and carbon flux of gluconeogenesis pathway and TCA cycle were reduced to enhance the production of acetone. Finally, resting cells biotransformation together with gas-stripping process was performed to further improve the production and recovery of acetone. The results showed a great potential to replace the fossil fuel based acetone manufacturing with biosynthesis from the renewable feedstock of acetate.

\section{Results and discussion}

Enhanced acetone biosynthesis from acetate through hybrid synthetic pathway from C. acetobulylicum and $E$. coli

In traditional acetone synthetic pathway, acetate is used as a receptor to accept the CoA from acetoacetyl-CoA. However, in E. coli, acetate is one of the main by-products from glucose metabolism. Here, we proposed to use acetate directly as the sole carbon source for acetone biosynthesis. In this pathway, acetate is first converted into acetyl-CoA through ACS (acetyl-CoA synthetase) pathway or ACK-PTA pathway. Then, 2 mol of acetyl$\mathrm{CoA}$ are condensed by thiolase to generate $1 \mathrm{~mol}$ of acetoacetyl-CoA. Acetoacetyl-CoA transferase transfers the CoA moiety from acetoacetyl-CoA to acetate, and forms acetoacetate and acetyl-CoA, which is part of endogenous acetate utilization pathway. Finally, acetoacetate is catalyzed to form acetone and carbon dioxide by acetoacetate decarboxylase (Fig. 1). Hence, the maximum theoretical yield of acetone is $0.5 \mathrm{~mol}$ acetone $/ \mathrm{mol}$ acetate. Taken from native acetone producing $C$. acetobutylicum, genes $t h l, a d c$ and $c t f A B$ were first cloned into pTrc99a to generate pTrcTAC. pTrcTAC was then introduced into E. coli MG1655 for acetone production. As thiolase $(t h l)$ and CoA transferase (ctfAB) each have endogenous alternatives in E. coli (atoB for thl, and atoDA for ctfAB), we further constructed three more plasmids (pTrcBAC, pTrcBAD and pTrcTAD) with different combinations of hybrid acetone biosynthesis pathways (Fig. 1). As shown in Fig. 2a, introduction of heterologous acetone biosynthesis pathway in MG1655 impaired the growth of recombinant strains compared to the control strain MG1655(pTrc99a). The cell growth defect suggested that central metabolic pathway did not function well possibly because of lack of enough metabolite precursors. MG1655(pTrc99a) consumed all acetate in $36 \mathrm{~h}$ with no acetone production (Fig. 2b, c). Meanwhile, the acetate consumption rate of $a t o B$ containing strains was relatively low, which indicated Thl was more effective than AtoB for acetate assimilation. MG1655(pTrcTAD) exhibited the highest consumption rate of acetate among the four strains, and the acetone accumulation reached $18.8 \mathrm{mM}$, which was about 6.5 times, 2.4 times and 2.1 times of that 


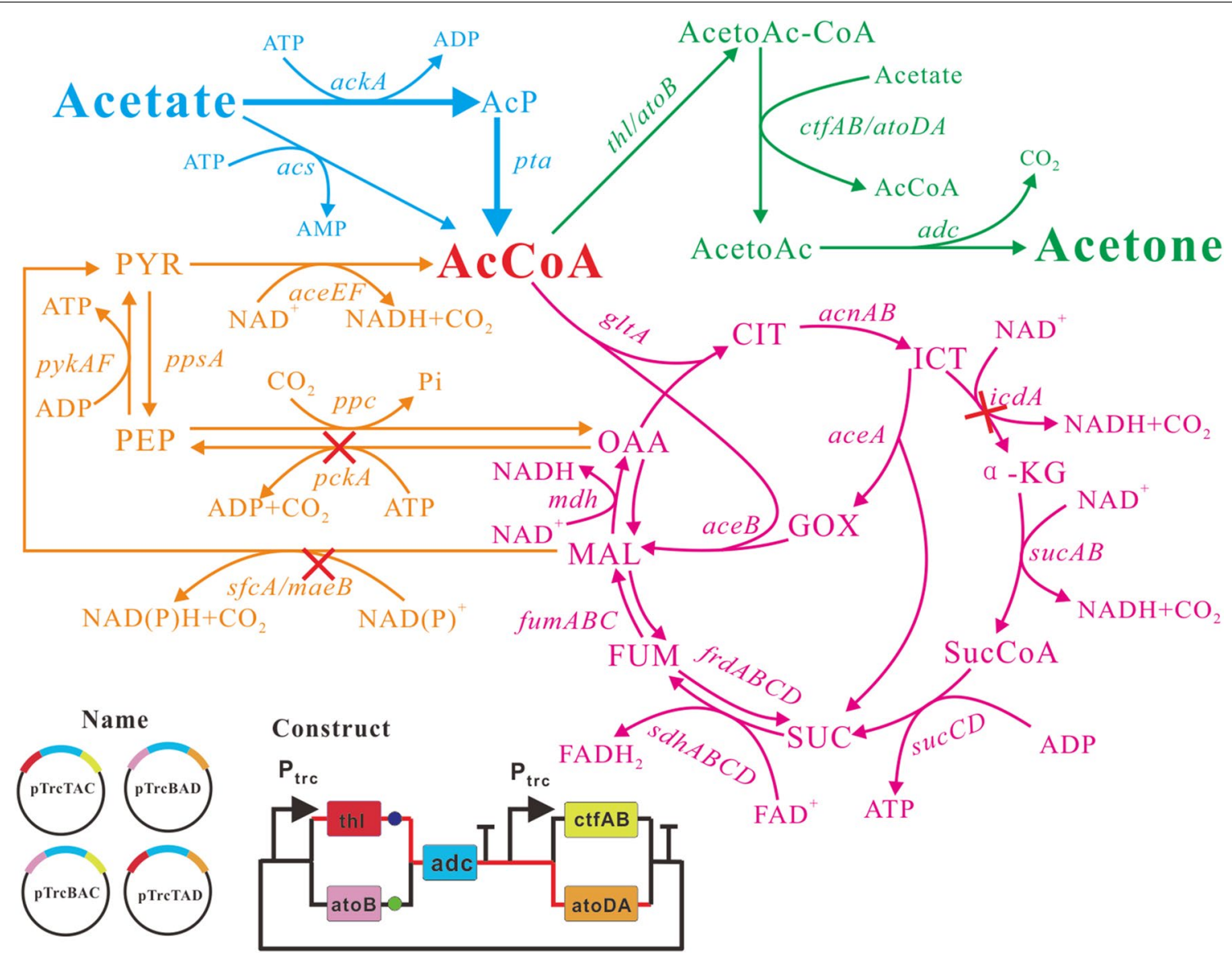

Fig. 1 Simplified metabolic pathways of acetone biosynthesis by engineered E. coli strain using acetate as carbon source under aerobic condition. Blue arrows represent acetate assimilation pathway, green arrows for acetone synthesis, orange arrows for futile cycle and purple arrows for tricarboxylic acid cycle. Four plasmids containing the acetone biosynthesis pathways were constructed and investigated

produced by MG1655(pTrcBAD), MG1655(pTrcTAC) and MG1655(pTrcBAC), respectively. In addition, the acetone yield of MG1655(pTrcTAD) was about 1.9 times that of MG1655(pTrcTAC) $(0.17 \mathrm{~mol} / \mathrm{mol}$ vs $0.09 \mathrm{~mol} /$ mol) (Fig. 4). The fact that MG1655(pTrcTAD) had a better performance than MG1655(pTrcTAC) for acetone synthesis may be attributed to AtoDA, which has a higher acetate affinity $(\mathrm{Km}=53.1 \mathrm{mM})$ than $\mathrm{CtfAB}$ $(\mathrm{Km}=1200 \mathrm{mM})$ [4]. This was consistent with previously reported result of isopropanol production [23]. After acetone biosynthesis pathway from acetate was constructed, the rate of acetate consumption and the acetone yield need to be further improved.

\section{Effect of enhancing the acetate up-take rate on acetone biosynthesis}

In $E$. coli, the precursor molecule, acetyl-CoA, can be generated not only from pyruvate via several pathways depending on the oxygen conditions, but also from long chains fatty acids and acetate [24]. In order to metabolize different concentrations of acetate to acetyl-CoA, two distinct routes including reversible ACK-PTA pathway (low affinity for acetate) and irreversible ACS pathway (high affinity for acetate) are both existed in E. coli [25]. In previous studies, single acs gene has been overexpressed to accelerate acetate assimilation [6, 26]. However, for high concentration of acetate, overexpression of acs has no significant effect on acetate utilization. In addition, less ATP will be consumed in ACK-PTA pathway due to formation of ADP rather than AMP [24]. Recently, the ACK-PTA pathway was chosen as the target to enhance acetate assimilation during the production of succinate [27] and polyhydroxyalkanoates [19] from acetate. In order to save energy and improve acetone production under high concentration of extracellular acetate, we chose to engineer the ACK-PTA pathway to enhance acetate utilization. In this study, a modified Ptrc promoter (Additional file 1: Table S2) was used to replace the native promoter which was shared by ack and pta genes in E. coli MG1655, yielding strain HY01. The 

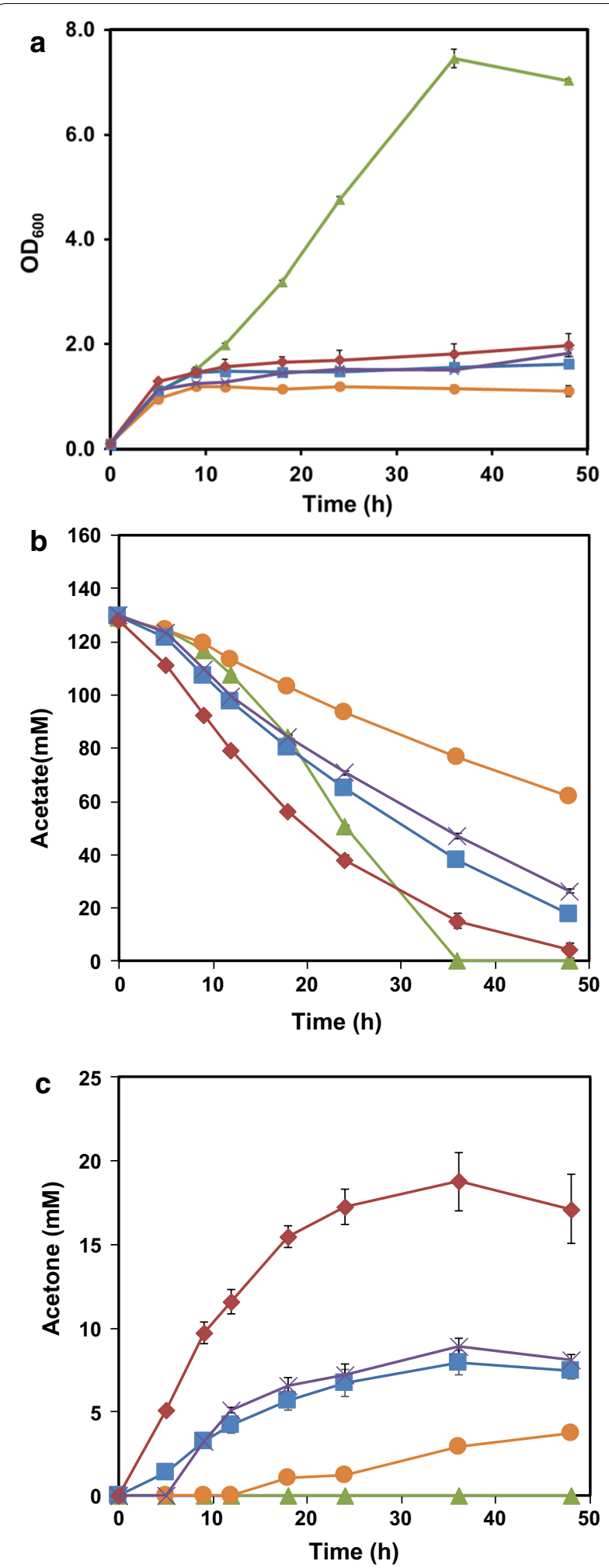

Fig. 2 Profiles of cell density (a), acetate (b) and acetone (c) concentrations in cultivation of different strains: MG1655(pTrc99a) (green triangle), MG1655(pTrcBAD) (orange circle), MG1655(pTrcBAC) (x), MG1655(pTrcTAC) (blue square), MG1655(pTrcTAD) (red diamond) cell growth of the engineered strain was recovered compared to the control one (Fig. 3a). The acetate consumption of HY01(pTrcTAD) in $24 \mathrm{~h}$ was about $12.9 \%$ higher than that of MG1655(pTrcTAD) (102.3 mM vs $90.6 \mathrm{mM})$ (Fig. 3b). Meanwhile, the acetone accumulation of HY01(pTrcTAD) reached 1.22 times of that produced by MG1655(pTrcTAD) (21.2 mM vs $17.3 \mathrm{mM}$ ) (Fig. 3c). The yield of HY01(pTrcTAD) was increased by $8.4 \%$ comparing with that of MG1655(pTrcTAD) $(0.21 \mathrm{~mol} / \mathrm{mol}$ vs $0.19 \mathrm{~mol} / \mathrm{mol}$ ) (Fig. 4). It still can be improved toward the maximum theoretical yield. These results indicated that the enhancement of ACK-PTA pathway was beneficial to acetate utilization and acetone biosynthesis.

\section{Effect of deletion of PCK and ME on acetone biosynthesis}

Blockage of undesired pathway is a common strategy to reduce carbon loss in metabolic engineering. In our previous study, deletion of $p c k A$ and maeB improved succinate production when acetate was used as carbon source [27]. It is also proved that blocking the pathway toward gluconeogenesis can theoretically reduce $\mathrm{CO}_{2}$ emission and activate glyoxylate shunt [28]. As is known, phosphoenolpyruvate carboxykinase $(p c k A)$ is responsible for converting oxaloacetate (OAA) to phosphoenolpyruvate (PEP) which releases $\mathrm{CO}_{2}$ and consumes ATP. $\mathrm{NADP}^{+}$-dependent malic enzyme (maeB) catalyzes malate into pyruvate with $\mathrm{CO}_{2}$ forming, too. Meanwhile, these two reactions can also drive carbon flux from TCA cycle to gluconeogenesis pathway. Therefore, $p c k A$ and maeB were deleted in strain HY01, forming HY021 and HY022, respectively. HY031 was further constructed by deletion of maeB in HY021. The growth of strain HY021(pTrcTAD) was slightly impaired, while the growth of maeB mutant strains were improved significantly. HY022(pTrcTAD) and HY031(pTrcTAD) grew better in acetate than the control strain HY01(pTrcTAD), which were about $26 \%$ and $11.3 \%$ higher than that of HY01(pTrcTAD), respectively (Fig. 3a). In the meantime, it was interesting that the consumptions of acetate consumption and acetone production among these three engineered strains were similar with the HY01(pTrcTAD) (Fig. 3b), which indicated that the growth yield of the meaB mutant strains were higher than that of HY01(pTrcTAD), and less carbon were lost in the form of $\mathrm{CO}_{2}$. Moreover, the yield of HY031(pTrcTAD) was slightly higher than HY022(pTrcTAD). Thus, HY031 was chosen to be further modified for better performance.

\section{Improving the acetone yield by deletion of icdA}

TCA cycle is one of the center metabolism pathway and plays a significant role in supplement of energy and intermediate metabolites for cell metabolism. The energy generated from TCA cycle is essential for acetate 

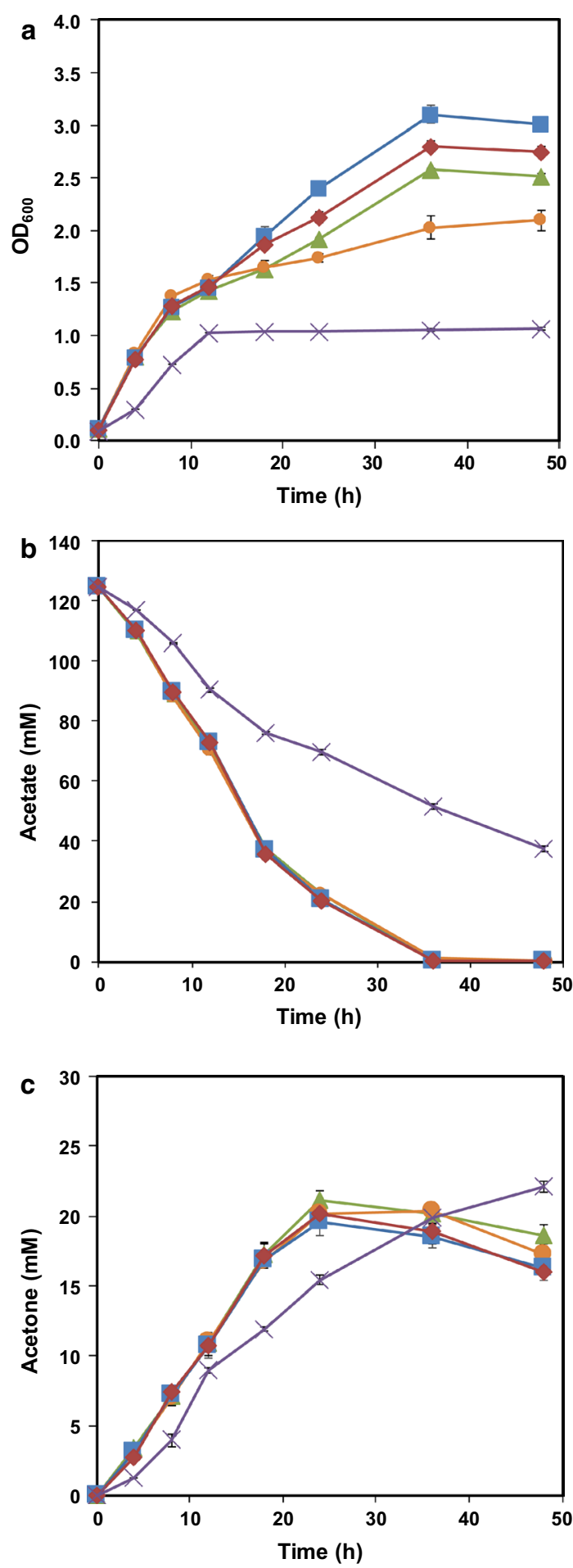

Fig. 3 Profiles of cell density (a), acetate (b) and acetone (c) concentrations in cultivation of different strains: HY01 (pTrcTAD) (green triangle), HY021 (pTrcTAD) (orange circle), HY022(pTrcTAD) (blue square), HY031 (pTrcTAD) (red square), HY041 (pTrcTAD) (x) 


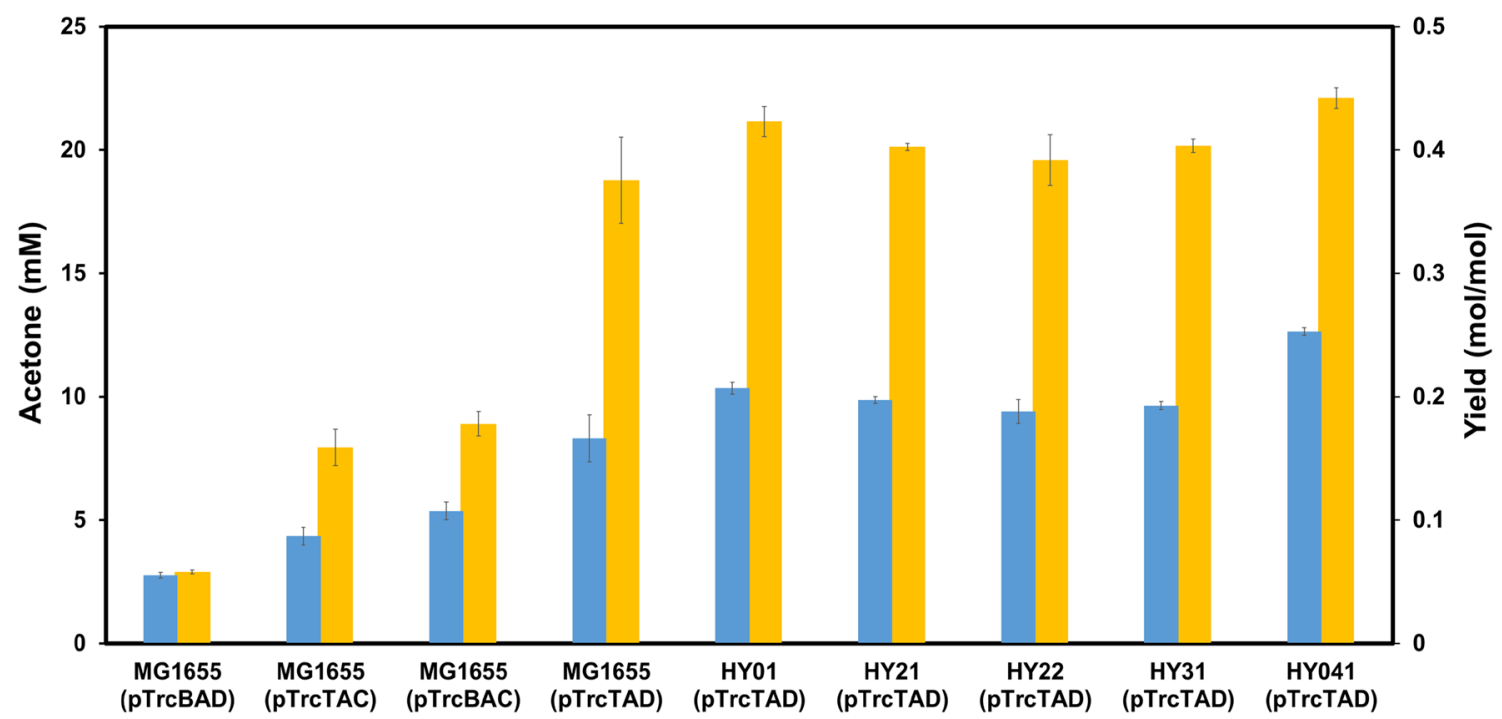

Fig. 4 Acetone production and the yield of different metabolically engineered E. coli strains in different conditions (yellow square) the titer of acetone; (blue square) the yield of acetone on acetic acid

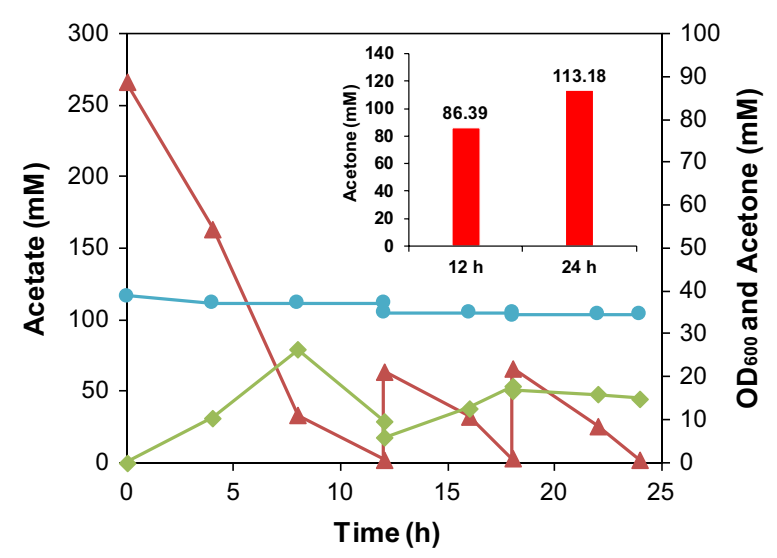

Fig. 5 Profiles of cell density (blue circle), acetate (red triangle), and acetone (green triangle) concentrations in the resting cell system of HY041 (pTrcTAD) with high cell density $\left(\sim 35 \mathrm{OD}_{600}\right)$

acetone was produced with a yield of $0.42 \mathrm{~mol}$ acetone/ $\mathrm{mol}$ acetate in $24 \mathrm{~h}$ because extra yeast extract was added (Additional file 1: Fig. S2). After 24 h' cultivation, the cells were harvested for resting cells biotransformation. After $24 \mathrm{~h}$ of biotransformation, almost all of the acetate (about $390 \mathrm{mM}$ ) was consumed and $113.18 \mathrm{mM}$ of acetone was obtained, with the yield of $0.29 \mathrm{~mol}$ acetone $/ \mathrm{mol}$ acetate (Fig. 5, Additional file 1: Fig. S3). The titer of acetone in the resting cell biotransformation was close to the concentration obtained by using glucose fed-batch cultures, which produced $122 \mathrm{mM}$ acetone in $48 \mathrm{~h}$ in E. coli [5]. Meanwhile, our results exceeded the titer of acetone achieved in traditional $\mathrm{ABE}$ fermentation with $C$. acetobutylicum [32].

\section{Conclusions}

In this study, hybrid biosynthesis pathway using acetate as sole carbon source was constructed by expressing various combinations of genes from $C$. acetobutylicum and $E$. coli. The strain with the combination of atoDA from E. coli and thl and adc from C. acetobulylicum exhibited the highest titer and yield of acetone. To further improve acetate assimilation and acetone production, a serial of genetic manipulations were conducted to engineer the host $E$. coli. The enhancement of ACK-PTA pathway by exchanging promoter of -ack-pta improved the acetate assimilation and further increased acetone production significantly. Blocking of gluconeogenesis pathway (deletion of gene $p c k A$ and gene $m a e B$ ) was proved to have no effect on acetate assimilation but played an important role on cell growth. The redirection of carbon flux into glyoxylate shunt (deletion of gene $i c d A$ ) dramatically improved the yield through bypassing the carbon emission reactions in the oxidative branch of TCA cycle. The engineered $E$. coli strain HY041(pTrcTAD) produced $22.1 \mathrm{mM}$ acetone with the yield of $0.25 \mathrm{~mol}$ acetone $/ \mathrm{mol}$ acetate in $48 \mathrm{~h}$. In the resting cell experiment under high cell density with gas-stripping technology, strain HY041 (pTrcTAD) produced $113.18 \mathrm{mM}$ acetone with the yield increased to $0.29 \mathrm{~mol}$ acetone $/ \mathrm{mol}$ acetate in $24 \mathrm{~h}$. These results suggested great potential of these engineered strains for industrial production of acetone from acetate. 


\section{Methods}

\section{Strains and plasmids}

A list of strains and plasmids used are shown in Table 1. Detailed primers for the construction of strains and plasmids are listed in Additional file 1: Table S1. Modified trc promotor (Ptrc1, Additional file 1: Table S2) was substituted for the native promotor of ackA-pta in host strain E. coli MG1655 by the one-step PCR-targeting method [33] as same as the following deletions of chromosomal $p c k A$, maeB and $i c d A$. For the gene deletion in E. coli, the DNA fragment containing the kanamycin resistance cassette and homologous arm of recombination was amplified by PCR using the genomic DNA from a single deleted strain (JW3366-1 for deletion of pckA, JW2447-5 for deletion of maeB and JW1122-2 for deletion of $i c d A$ ) as template.

The construction of recombinant plasmids were described as followed. The genome of E. coli MG1655 and Clostridium acetobulylicum were used as the PCR templates. Gene segments of ato $B$, ato $D A$ from E. coli and thl, adc from Clostridium acetobulylicum were amplified by PCR. The sequence of lacI terminator along with the trc promoter in the plasmid pTrc99a was amplified as a trc promoter cassette by PCR. These resulting DNA fragments were overlapped together to form $\operatorname{trc}$-atoDA, $\operatorname{trc}-\mathrm{ctf} A B$, atoB-RBS-adc and thl-RBSadc (Additional file 1: Table S3). The optimized RBS between $a t o B-a d c$ and $t h l$-adc were calculated by using online software (https://salislab.net/software/forward). The DNA fragments, trc-atoDA, trc-ctfAB, were ligated into linear vector pTrc99a which was digested by Pst I and Hind III to form pTrc99a-trc-atoDA and pTrc99a$\operatorname{trc}-\operatorname{ctf} A B$, respectively. Then, the DNA fragment of ato $B$ RBS- $a d c$ was cloned into the plasmid pTrc99a-trc-atoDA to form pTrc99a-atoB-RBS-adc-trc-atoDA (pTrcBAD). The DNA fragment of $t h l-\mathrm{RBS}-a d c$ was cloned into the plasmid pTrc99a-trc-atoDA to form pTrc99a-thl-RBSadc-Trc-atoDA (pTrcTAD). The DNA fragment of atoBRBS-adc was cloned into the plasmid pTrc99a-trc-ctfAB to form pTrc99a-atoB-RBS-adc-trc-ctfAB (pTrcBAC). The DNA fragment of thl-RBS-adc was cloned into the plasmid pTrc99a-trc-ctfAB to form pTrc99a-thl-RBSadc-trc-ctfAB (pTrcTAC). HB-infusion ${ }^{\mathrm{TM}}$ Master mix from Hanbio (Shanghai, China) was used for the above recombination plasmids construction via seamless ligation cloning. PrimeSTAR ${ }^{\circledR}$ HS DNA Polymerase was purchased from Takara (Dalian, China). Restriction enzymes were purchased from NEB (Beijing, China). All resulted plasmids were confirmed by DNA sequencing by Sangon Company (Shanghai, China).

\section{Table 1 Strains and plasmids}

\begin{tabular}{|c|c|c|}
\hline Strains and plasmids & Relevant characteristics & Source \\
\hline \multicolumn{3}{|l|}{ Strains } \\
\hline E. coli MG1655 & Wild type & Laboratory collection \\
\hline $\begin{array}{l}\text { Clostridium acetobulylicum } \\
\text { ATCC } 184\end{array}$ & Wild type & ATCC \\
\hline JW3366-1 & BW25113, $\Delta p c k A:: \mathrm{Km}^{\mathrm{R}}$ & Coli genetic stock center (CGSC) \\
\hline JW2447-5 & BW25113, $\Delta$ meaB:: $\mathrm{Km}^{\mathrm{R}}$ & Coli genetic stock center (CGSC) \\
\hline$J W 1122-2$ & BW25113, $\triangle i c d A:: \mathrm{Km}^{\mathrm{R}}$ & Coli genetic stock center (CGSC) \\
\hline HY01 & MG1655, Ptrc-ackA-pta & This study \\
\hline HY021 & MG1655, Ptrc-ackA-pta, $\Delta p c k A$ & This study \\
\hline HY022 & MG1655, Ptrc-ackA-pta, $\triangle m a e B$ & This study \\
\hline HY031 & MG1655, Ptrc-ackA-pta, $\Delta p c k A, \triangle m a e B$ & This study \\
\hline HY041 & MG1655, Ptrc-ackA-pta, $\Delta p c k A, \triangle m a e B, \Delta i c d A$ & This study \\
\hline \multicolumn{3}{|l|}{ Plasmids } \\
\hline pKD4 & oriR6Kץ, Km ${ }^{R}, r g n B(T e r)$ & Datsenko and Wanner [33] \\
\hline pKD46 & araBp-gam-bet-exo, bla $\left(\mathrm{Ap}^{\mathrm{R}}\right)$, repA101 (ts), oriR101 & Datsenko and Wanner [33] \\
\hline pCP20 & $A p^{R}, C m^{R}, F L P$ recombinance & Datsenko and Wanner [33] \\
\hline pTrc99a & Cloning vector, trc promotor, $A p^{R}$ & Laboratory collection \\
\hline pTrc99a-trc-atoDA & pTrc99a carries trc promoter with atoD and atoA from E. coli & This study \\
\hline pTrc99a-trc-ctfAB & pTrc99a carries trc promoter with ctfA and ctfB from Clostridium acetobulylicum & This study \\
\hline pTrcBAD & pTrc99a-trc-atoDA carries atoB from E. coli and adc from Clostridium acetobulylicum & This study \\
\hline pTrcTAD & pTrc99a-trc-atoDA carries thl and adc from Clostridium acetobulylicum & This study \\
\hline pTrcBAC & pTrc99a-trc-ctfAB carries atoB from E. coli and adc from Clostridium acetobulylicum & This study \\
\hline pTrcTAC & pTrc99a-trc-ctfAB carries th/ and adc from Clostridium acetobulylicum & This study \\
\hline
\end{tabular}




\section{Media}

Luria-Bertani (LB) broth (per liter: tryptone $10 \mathrm{~g}$, yeast extract $5 \mathrm{~g}$, sodium chloride $10 \mathrm{~g}$ ) was used for the construction of strains and seeds culture. The M9 minimal salts medium consisted of the following components (per liter): $15.12 \mathrm{~g} \mathrm{Na}_{2} \mathrm{HPO}_{4} \cdot 12 \mathrm{H}_{2} \mathrm{O}, 0.5 \mathrm{~g} \mathrm{KH}_{2} \mathrm{PO}_{4}, 3.0 \mathrm{~g}$, $\mathrm{NaCl}, 0.5 \mathrm{~g} \mathrm{MgSO}_{4} \cdot 7 \mathrm{H}_{2} \mathrm{O}, 0.011 \mathrm{~g} \mathrm{CaCl}_{2}, 1.0 \mathrm{~g} \mathrm{NH}_{4} \mathrm{Cl}$, $0.2 \mathrm{~mL} 1 \%(\mathrm{w} / \mathrm{v})$ vitamin $\mathrm{B} 1$, and $0.1 \mathrm{~mL}$ trace elements solution. The stock solution of trace elements contained the following components (per liter) in $3 \mathrm{M} \mathrm{HCl}: 80 \mathrm{~g}$ $\mathrm{FeSO}_{4} \cdot 7 \mathrm{H}_{2} \mathrm{O}, 10 \mathrm{~g} \mathrm{AlCl}_{3} \cdot 6 \mathrm{H}_{2} \mathrm{O}, 2.0 \mathrm{~g} \mathrm{ZnSO}_{4} \cdot 7 \mathrm{H}_{2} \mathrm{O}, 1.0 \mathrm{~g}$ $\mathrm{CuCl}_{2} \cdot 2 \mathrm{H}_{2} \mathrm{O}, 2.0 \mathrm{~g} \mathrm{NaMoO} \cdot 2 \mathrm{H}_{2} \mathrm{O}, 10 \mathrm{~g} \mathrm{MnSO}_{4} \cdot \mathrm{H}_{2} \mathrm{O}$, $4.0 \mathrm{~g} \mathrm{CoCl}_{2}$, and $0.5 \mathrm{~g} \mathrm{H}_{3} \mathrm{BO}_{4}$. The SMAC media was a modified M9 medium containing $2 \mathrm{~g} / \mathrm{L}$ of yeast extract and $10 \mathrm{~g} / \mathrm{L}$ of sodium acetate. Appropriate antibiotics were included at the following concentrations: ampicillin, $100 \mathrm{mg} / \mathrm{L}$; kanamycin, $50 \mathrm{mg} / \mathrm{L}$; chloramphenicol, $34 \mathrm{mg} / \mathrm{L}$.

\section{Culture conditions}

A single colony from a freshly grown plate was inoculated in $3 \mathrm{~mL}$ of LB medium at $220 \mathrm{rpm}$ and $37^{\circ} \mathrm{C}$ for overnight culture. $1 \mathrm{~mL}$ of the primary preculture culture was inoculated $(2 \%, \mathrm{v} / \mathrm{v})$ into $50 \mathrm{~mL} \mathrm{LB}$ media for aerobic growth in 250-mL conical flask for $10 \mathrm{~h}$. The secondary preculture cultures were inoculated into SMAC media for shake flask fermentation at the ratio of $2 \%(\mathrm{v} / \mathrm{v})$. Fermentation was carried out at $37{ }^{\circ} \mathrm{C}, 220 \mathrm{rpm}$ until the $\mathrm{OD}_{600}$ reached around 1.0. The expression of the key enzymes of the hybrid acetone biosynthesis pathway were induced by the addition of isopropyl- $\beta$-D-thiogalactopyranoside (IPTG) to the final concentrations of $0.1 \mathrm{mM}$. The incubation temperature was adjusted to $25{ }^{\circ} \mathrm{C}$ after adding IPTG. The sterilized $\mathrm{H}_{2} \mathrm{SO}_{4}(3 \mathrm{M})$ was used to adjust the $\mathrm{pH}$ of cultures to 7.0 during the fermentation. The volatilization of different concentrations of acetone in flasks were detected (Additional file 1: Fig. S4). All experiments in shake flasks were performed in triplicate.

Resting cells fermentation was performed to increase the productivity of acetone from acetate, using the concentrated resting engineered $E$. coli strain HY041(pTrcTAD). In the resting cell experiments, the preculture conditions were the same as that of the shake-flask fermentation. The modified M9 minimal medium contained $5 \mathrm{~g} / \mathrm{L}$ yeast extract instead of $2 \mathrm{~g} / \mathrm{L}$ yeast extract was used for cell cultivation. After cell density $\left(\mathrm{OD}_{600}\right)$ reached about 1.5 , the cells were harvested by centrifugation at $6000 \mathrm{rpm}$ and $4{ }^{\circ} \mathrm{C}$ for $10 \mathrm{~min}$. The harvested cells were washed twice by the M9 medium without $\mathrm{NH}_{4} \mathrm{Cl}$. Then, cells pellets were resuspended to $100 \mathrm{~mL}$ of sterile $\mathrm{NH}_{4} \mathrm{Cl}$-free $\mathrm{M} 9$ medium containing $20 \mathrm{~g} / \mathrm{L}$ sodium acetate without adding trace elements and vitamin $\mathrm{B} 1$. The resuspended cell broth $\left(35 \mathrm{OD}_{600}\right)$ were transferred into a simplified mini-reactor with automatic $\mathrm{pH}$ control system (Additional file 1: Fig. S1). The $\mathrm{pH}$ was kept at 7.0 using $3 \mathrm{M} \mathrm{H}_{2} \mathrm{SO}_{4}$, and the temperature was maintained at $25{ }^{\circ} \mathrm{C}$. Due to the air supply of the minireactor, the effect of gas-tripping can be achieved during the resting cell experiments. Ten bottles of $100 \mathrm{~mL}$ sterilized $\mathrm{H}_{2} \mathrm{O}$ were connected sequentially and linked to the off-gas of the simplified mini-reactor for the acetone collection (Additional file 1: Figure S1). The total amounts of acetone were calculated at $24 \mathrm{~h}$ by combining the acetone in all of these bottles with the mini-reactor.

\section{Analytical methods}

Cell density was detected by measuring the optical density of appropriately diluted culture samples at $600 \mathrm{~nm}$ $\left(\mathrm{OD}_{600}\right)$. Culture samples were centrifuged for $10 \mathrm{~min}$ at $4{ }^{\circ} \mathrm{C}$ and $13,000 \times g$. The supernatant was then filtered through a $0.22 \mu \mathrm{m}$ nylon syringe filter. The concentrations of acetone and acetate were detected by HPLC with an aminex HPX-87H ion exclusion column (Bio-Rad, USA), a refractive index detector (RID-10A, Shimadzu Corporation, Kyoto, Japan), a UV detector (SPD-10A, Shimadzu Corporation, Kyoto, Japan), an on-line degasser system (DGU-20A3; Shimadzu) and an LC Solutions system (Shimadzu Corporation, Kyoto, Japan). The mobile phase was $2.5 \mathrm{mM} \mathrm{H}_{2} \mathrm{SO}_{4}$ running at $0.5 \mathrm{~mL} / \mathrm{min}$, and the column temperature was operated at $50{ }^{\circ} \mathrm{C}$.

\section{Additional file}

Additional file 1. Additional tables and figures.

\section{Abbreviations}

ABE fermentation: acetone-butanol-ethanol fermentation; ACK-PTA: acetate kinase and phosphotransacetylase; ACS: acetyl-CoA synthetase; ADP: adenosine diphosphate; AMP: adenosine monophosphate; ATP: adenosine triphosphate; IPTG: isopropyl- $\beta$-D-thiogalactopyranoside; OAA: oxaloacetate; OD: optical density; RBS: ribosome binding site; TCA: tricarboxylic acid cycle.

\section{Authors' contributions}

$\mathrm{HW}$ designed the experiments; $\mathrm{HY}$ and $\mathrm{BH}$ performed the research experiments; NL and YG helped in the experiments; $\mathrm{QY}$ and ZL provided technical support; $\mathrm{HY}, \mathrm{BH}$, and $\mathrm{HW}$ analysis the data; $\mathrm{HY}, \mathrm{BH}$, and $\mathrm{HW}$ wrote the manuscript. All authors read and approved the final manuscript.

\section{Author details \\ ${ }^{1}$ State Key Laboratory of Bioreactor Engineering, East China University of Sci- ence and Technology, 130 Meilong Road, Shanghai 200237, China. ${ }^{2}$ Shang- hai Collaborative Innovation Center for Biomanufacturing Technology, 130 Meilong Road, Shanghai 200237, China. ${ }^{3}$ Key Laboratory of Bio-based Material Engineering of China National Light Industry Council, 130 Meilong Road, Shanghai 200237, China. ${ }^{4}$ Key Laboratory of Synthetic Biology, CAS Center for Excellence in Molecular Plant Sciences, Shanghai Institute of Plant Physiol- ogy and Ecology, Chinese Academy of Sciences, Shanghai 200032, China.}

\section{Acknowledgements}

We thank for Dr. Yipeng Wang for helpful editing and comments on the manuscript. 


\section{Competing interests}

The authors declare that they have no competing interests.

\section{Availability of data and materials}

All data generated or analyzed during this study are included in this published article and its additional files

\section{Consent for publication}

Not applicable.

\section{Ethics approval and consent to participate} Not applicable.

\section{Funding}

This study was supported by the National Natural Science Foundation of China (Grant No. 21776083), the Fok Ying-Tong Education Foundation, China (Grant No. 161017), the National Key Research and Development Program of China (Grant No. 2017YFB0309302), the Science and Technology Commission of Shanghai Municipality (Grant No. 17JC1404800), the Fundamental Research Funds for the Central Universities (Grant No. 22221818014). Partially supported by Open Funding Project of the State Key Laboratory of Bioreactor Engineering.

\section{Publisher's Note}

Springer Nature remains neutral with regard to jurisdictional claims in published maps and institutional affiliations.

Received: 14 November 2018 Accepted: 7 January 2019

Published online: 14 January 2019

\section{References}

1. Schmidt RJ. Industrial catalytic processes - phenol production. Appl Catal A. 2005:280:89-103.

2. Gu Y, Jiang Y, Yang S, Jiang W. Utilization of economical substrate-derived carbohydrates by solventogenic clostridia: pathway dissection, regulation and engineering. Curr Opin Biotechnol. 2014;29:124-31.

3. Liao C, Seo SO, Celik V, Liu H, Kong W, Wang Y, Blaschek H, Jin YS, Lu T. Integrated, systems metabolic picture of acetone-butanol-ethanol fermentation by Clostridium acetobutylicum. Proc Natl Acad Sci USA. 2015:112:8505-10.

4. Bermejo LL, Welker NE, Papoutsakis ET. Expression of Clostridium acetobutylicum ATCC 824 genes in Escherichia coli for acetone production and acetate detoxification. Appl Environ Microbiol. 1998;64:1079-85.

5. May A, Fischer RJ, Maria TS, Schaffer S, Verseck S, Dürre P, Bahl H. A modified pathway for the production of acetone in Escherichia coli. Metab Eng. 2013;15:218-25.

6. Yang X, Yuan Q, Zheng Y, Ma H, Chen T, Zhao X. An engineered nonoxidative glycolysis pathway for acetone production in Escherichia coli. Biotechnol Lett. 2016;38:1359-65.

7. Jiang J, Huang B, Wu H, Li Z, Ye Q. Efficient 3-hydroxypropionic acid production from glycerol by metabolically engineered Klebsiella pneumoniae. Bioresour Bioprocess. 2018;5:34.

8. Li Q, Huang B, He Q, Lu J, Li X, Li Z, Wu H, Ye Q. Production of succinate from simply purified crude glycerol by engineered Escherichia coli using two-stage fermentation. Bioresour Bioprocess. 2018:5:41.

9. Conrado RJ, Gonzalez R. Envisioning the bioconversion of methane to liquid fuels. Science. 2014;343:621-3.

10. Sonntag F, Kroner C, Lubuta P, Peyraud R, Horst A, Buchhaupt M, Schrader J. Engineering Methylobacterium extorquens for de novo synthesis of the sesquiterpenoid a-humulene from methanol. Metab Eng. 2015;32:82-94.

11. Revelles O, Tarazona N, Garcia JL, Prieto MA. Carbon roadmap from syngas to polyhydroxyalkanoates in Rhodospirillum rubrum. Environ Microbiol. 2016;18:708-20.

12. Budiman AW, Ji SN, Park JH, Mukti RI, Chang TS, Bae JW, Choi MJ. Review of acetic acid synthesis from various feedstocks through different catalytic processes. Catal Surv Asia. 2016;20:1-21.
13. Munasinghe PC, Khanal SK. Biomass-derived syngas fermentation into biofuels: opportunities and challenges. Bioresour Technol. 2010;101:5013-22

14. Sakimoto KK, Wong AB, Yang PD. Self-photosensitization of nonphotosynthetic bacteria for solar-to-chemical production. Science. 2016;351:74-7.

15. Hu P, Chakraborty S, Kumar A, Woolston B, Liu H, Emerson D, Stephanopoulos $\mathrm{G}$. Integrated bioprocess for conversion of gaseous substrates to liquids. Proc Natl Acad Sci USA. 2016;113:3773.

16. Zhu Z, Sathitsuksanoh N, Vinzant T, Schell DJ, McMillan JD, Zhang YHP. Comparative study of corn stover pretreated by dilute acid and cellulose solvent-based lignocellulose fractionation: enzymatic hydrolysis, supramolecular structure, and substrate accessibility. Biotechnol Bioeng. 2009;103:715-24

17. Liu JF, Shonnard DR. Life cycle carbon footprint of ethanol and potassium acetate produced from a forest product wastewater stream by a colocated biorefinery. ACS Sustain Chem Eng. 2014;2:1951-8.

18. Eerten-Jansen MC, Heijne AT, Grootscholten TI, Steinbusch KJ, Sleutels $\mathrm{TH}$, Hamelers HV, Buisman CJ. Bioelectrochemical production of caproate and caprylate from acetate by mixed cultures. ACS Sustain Chem Eng. 2013;1:513-8.

19. Lee HM, Jeon BY, Oh MK. Microbial production of ethanol from acetate by engineered Ralstonia eutropha. Biotechnol Bioprocess Engin. 2016;21:402-7

20. Noh MH, Lim HG, Woo SH, Song J, Jung GY. Production of itaconic acid from acetate by engineering acid-tolerant Escherichia coli W. Biotechnol Bioeng. 2018;115:729-38.

21. Chen J, Li W, Zhang ZZ, Tan TW, Li ZJ. Metabolic engineering of Escherichia coli for the synthesis of polyhydroxyalkanoates using acetate as a main carbon source. Microb Cell Fact. 2018;17:102.

22. Xu X, Xie M, Zhao Q, Xian M, Liu H. Microbial production of mevalonate by recombinant Escherichia coli using acetic acid as a carbon source. Bioengineered. 2018;9:116-23.

23. Hanai T, Atsumi S, Liao JC. Engineered synthetic pathway for isopropanol production in Escherichia coli. Appl Environ Microbiol. 2007;73:7814-8.

24. Krivoruchko A, Zhang Y, Siewers V, Chen Y, Nielsen J. Microbial acetyl-CoA metabolism and metabolic engineering. Metab Eng. 2015;28:28-42.

25. Starai VJ, Escalante-Semerena JC. Acetyl-coenzyme A synthetase (AMP forming). Cell Mol Life Sci. 2004;61:2020-30.

26. Lin H, Castro NM, Bennett GN, San KY. Acetyl-CoA synthetase overexpression in Escherichia coli demonstrates more efficient acetate assimilation and lower acetate accumulation: a potential tool in metabolic engineering. Appl Microbiol Biotechnol. 2006;71:870-4.

27. Huang B, Yang H, Fang GC, Zhang X, Wu H, Li ZM, Ye Q. Central pathway engineering for enhanced succinate biosynthesis from acetate in Escherichia coli. Biotechnol Bioeng. 2018;115:943-54.

28. Yang CHQ, Baba T, Mori H, Shimizu K. Analysis of Escherichia coli anaplerotic metabolism and its regulation mechanisms from the metabolic responses to altered dilution rates and phosphoenolpyruvate carboxykinase knockout. Biotechnol Bioeng. 2003;84:129-44.

29. Li Y, Huang B, Wu H, Li Z, Ye Q, Zhang YP. Production of succinate from acetate by metabolically engineered Escherichia coli. Acs Synthetic Biol. 2016;5:1299-307

30. Ferguson $\mathrm{BJ}$, Indrasumunar A, Hayashi S, Lin MH, Lin YH, Reid DE, Gresshoff PM. Molecular analysis of legume nodule development and autoregulation. J Integr Plant Biol. 2010;52:61-76.

31. Apostolakos D, Menter PA, Rampsch BJ, Reeves HC, Birge EC. Genetic map position of the cistron coding for isocitrate dehydrogenase in Escherichia coli K-12. Curr Microbiol. 1982;7:45-7.

32. Bahl H, Gottschalk G. Microbial production of butanol/acetone. In: Rehm HJ, Reed G, editors. Biotechnology: a comprehensive treatise in 8 volumes, vol. 6b. Weinheim: VCH Verlagsgesellschaft; 1988. p. 1-30.

33. Datsenko KA, Wanner BL. One-step inactivation of chromosomal genes in Escherichia coli K-12 using PCR products. Proc Natl Acad Sci USA. 2000:97:6640-5. 